\title{
New Unknown Sars-Cov-2 Virus Variants and Hidden Pandemics Within Them in Developing Countries
}

\section{Shohreh Ghasemi}

Augusta University Medical College of Georgia

Mahmood Dashti ( $\sim$ dashti.mahmood72@gmail.com )

private practice at med spa https://orcid.org/0000-0003-2013-0374

\section{Short Report}

Keywords: sequencing, pandemic, Sars-COV2, Contamination, developing countries.

Posted Date: September 21st, 2021

DOl: https://doi.org/10.21203/rs.3.rs-898454/v1

License: (c) (1) This work is licensed under a Creative Commons Attribution 4.0 International License.

Read Full License 


\section{Abstract}

It is not known whether one or a combination of different mutations will alter the viral clinical and epidemiological manifestations, transmissibility, virulence, or efficacy of the vaccine. Transmission of the new variant by asymptomatic carriers is also unknown. Vaccines or antiviral agents have not yet induced the pressure of the mutation; however, other mutations are anticipated after global vaccination and after the introduction of proven therapies. Thus, a willingness to rapidly emerge new options is prudent. Less virulent but highly heritable variants can also be expected, which may contribute to herd immunity. There is a need to develop clinical and rapid laboratory tests to monitor vaccinated individuals for secondary infection potentially caused by the new variant. Importantly, restrictive countermeasures, spatial distancing, personal hygiene, travel bans, and facial disguises remain relevant in the fight against the virus.

\section{Introduction}

Covid-19 has crippled humanity over the past year, with all quarantines, deaths and isolation. However, with the advent of new vaccines like Oxford AstraZeneca, Moderna, Pfizer etc., there is hope that this horror will be over. However, all viruses develop over time as the virus copies or replicates itself; sometimes, it changes slightly, which is typical for a virus. These alterations are called "mutations." A virus with one or more new mutations is called a "variation" of the novel virus. When a virus circulates widely in a population and causes multiple infections, the chance of the virus transforming increases. The more opportunities a virus has for spreading, the more opportunities and more of its replicates have for changes (1). Multiple variants of SARS-CoV-2 circulate the world. In the fall of 2020, several new options appeared, primarily: (2)

A new variant of SARS-CoV-2 (known as 20I/501Y.V1, VOC 202012/01, or B.1.1.7) with a large number of mutations has appeared in the UK. Since then, this variant has been found in many countries worldwide, including the United States (USA). In28 January 2021, scientists from the UK presented evidence (3) showing that option B.1.1.7 may be associated with an increased risk of death than other options. More research is needed to confirm this conclusion. This new form was reported in the United States at the end of December 2020.

In South Africa, another modified form of SARS-CoV-2 (20H/501Y.V2 or B.1.351) arose independently of B.1.1.7. This variant has some transformations with B.1.1.7. Cases associated with this option have been found in many countries outside of South Africa. This option was reported in the United States at the end of January 2021.

In Brazil, the SARS-CoV-2 variant (known as P.1) was first identified in four travelers of Brazil who were tested in the course of a routine survey at Haneda Airport outside Tokyo, Japan. This modified form has 17 specific mutations and three in the spike protein of the receptor-binding domain. This variant was discovered in the United States at the end of January 2021(2-4). 
More about the variants

\section{Alpha: B.1.1.7 lineage (a.k.a. 20I/501Y.V1 Variant of Concern (VOC) 202012/01)}

This variation has a change in the receptor-binding domain (RBD) of the spike protein at position 501, where the corrosive amino asparagine $(N)$ is supplanted with tyrosine $(Y)$. The shortening for this change is N501Y. This variation additionally has a few different changes, including:

- This variant is believed to have first appeared in the UK in September 2020.

- Since 20 December 2020, several countries, including the United States, have reported lineage B.1.1.7.

- This option is associated with increased permeability (i.e., faster and more efficient Transmission).

- In January 2021, UK scientists presented evidence (3) showing that option B.1.1.7 may be associated with an increased risk of death compared to other options.

- Early reports found no evidence that this option in any way affects the severity of the disease or the effectiveness of the vaccine $(4,5,6)$

\section{Beta: B.1.351 lineage (a.k.a. 20H/501Y.V2)}

- This variant has several mutations in the spike protein, including E484K, K417N, N501Y. Unlike lineage B.1.1.7 found in the UK, this variant does not contain a 69/70 deletion.

- This variation was first perceived in Nelson Mandela Bay, South Africa, in preliminaries dating from early October 2020, and cases have since been found external South Africa, including the United States.

- An option was also identified in Zambia at the end of December 2020, when it proved to be the predominant option in the country.

- There is currently no evidence that this option in any way affects the severity of the disease.

- There is some evidence that one of the spike protein mutations, $\mathrm{E} 484 \mathrm{~K}$, may interfere with neutralization by some monoclonal and polyclonal antibodies $(6,7)$.

\section{Gamma: P.1 lineage (a.k.a. 20J/501Y.V3)}

- Option P.1 is an offshoot of line B.1.1.28, first reported by the National Institute of Infectious Diseases (NIID) in Japan from four Brazilian travelers who were sampled during routine screening at Haneda Airport outside Tokyo.

- Line P.1 contains three spike protein receptor-binding domain mutations: N501Y, K417T, and E484K.

- There is evidence to suggest that some mutations in variant P.1 may affect its transmissibility and antigenic profile, which may affect the ability of antibodies from previous natural infection or vaccination to recognize and neutralize the virus. 
- A new report revealed many cases in Manaus, the biggest city in the Amazon locale, wherein variation P.1 was recognized in $42 \%$ of tests sequenced since late December. (7) It is assessed that as of October 2020 , roughly $75 \%$ of the populace in this area has been contaminated with SARSCoV2. In any case, since mid-December, the area has seen an increment in the number of cases. The development of this variation raises worries about the likely expansion in contagiousness or the inclination of people to re-contamination with SARS-CoV-2.

- This new mutant was identified in the United States at the end of January 2021.

\section{Delta: B.1.617.2 Linage}

- Firstly, this variant in December 2020 in India (8) and after that prevailed with high peak in the United Kingdom (9).

- Delta variants are characterized by mutations in the protein T19R, T478K, $\Delta 157-158, \mathrm{~L} 452 \mathrm{R}, \mathrm{P} 681 \mathrm{R}$, D950N, and D614G(10). These mutations can affect the immune response directed to deletions of the significant antigenic regions (452 and 478) of receptor-binding proteins and some of the Nterminal domains (11). The P681R is located at the S1-S2 cut site, and strains with mutations appear to have increased replication, increasing viral load and infection rate (12).

- Bernal et al. revealed that the efficacy of the vaccine (BNT162b2 or ChAdOx1 nCoV-19) after a single dose was significantly lower among people with the delta variant $(30.7 \%)$ than with the alpha variant (48.7\%). Results were almost the same for both vaccines. With the BNT162b2 vaccine, the efficacy of double doses was $93.7 \%$ in patients with the alpha mutant and $88.0 \%$ in patients with the delta mutant. With the ChAdOx1 nCoV-19 vaccine, the efficacy of the two doses was $74.5 \%$ in alpha mutants and $67.0 \%$ in delta variants (9).

\section{Lambda: C.37 lineage}

- The lambda variant (C.37) was first reported in Peru in December 2020. Genome sequencing of the mutant revealed a deletion 1a (ORF1a) of the wild-type open reading frame ( $\Delta 3675-3677)$. Lambda variants also have new deletions in the $S$ protein $(\Delta 246-252)$ and multiple non-synonymous variants (L452Q, G75V, T76I, D614G, F490S, and T859N). The L452Q and F490S mutations are present in RBD spikes. The F490S mutation is associated with reduced susceptibility to antibody neutralization. There are 19 mutations in this variant, making it more contagious or resistant to antibodies induced by vaccination or previous exposure to the virus $(13,14,15)$.

- According to Acevedo et al., greater infectivity mediated by lambda peplomer was observed than D614G (line B) or alpha and gamma mutants. Moreover, neutralization was reduced by 3.05 times in the lambda variant, 2.33 times in the gamma variant, and 2.03 times in the alpha variant (15). The Tada et al. showed an average decrease of 2.3 to 3.3 times in antibody titer against lambda mutants (14).

\section{Mu: B.1.621 lineage}


- On August 30, 2021, it was categorized as a variation of interest (VOI) and given the WHO name "Mu."

- Although the global prevalence of the Mu variation among sequenced cases has decreased to below 0.1 percent, the prevalence in Colombia (39 percent) and Ecuador (40 percent) is still high (13 percent)

Despite a few transformations in spike,SARS-COV-2B.1.621 is neautarzalized by the Pfizer vaccine elicited antibodies, They note that the balance, in spite of the fact successful, was lower than that of the other corona virus variations(17).Snell et al ,proposes that MU variation has two cases of potential vaccine-espcape". They categorized in that a few of the spike changes inside Mu "have been detilaled to appear dimished balance by antibidoes .

The Mu variation was moreover found to have the same spike difeerentiation that has been delibilated immunization reaction with the Beta Variants. The presence of transformation related with immunisation elude might warrant renaming of this variation to a variation of concern (18)

\section{Other variations}

- The Eta variant (B.1.525) was first identified in Nigeria in December 2020. Its spike mutations include D614G, E484K, and Q677H, which affect antibody neutralization and transmission (19).

- Epsilon mutants (B.1.427 / B.1.429) were first identified in the United States in September 2020 and showed L452R, D614G as spike mutations, but their effect on antibody neutralization and transmission (19).

- Theta variant (P.3) was first identified in the Philippines in January 2021, and E484K, D614G, N501Y, and $\mathrm{P} 681 \mathrm{H}$ were detected as peak mutations affecting antibody neutralization and transmission (19).

- The kappa mutant (B.1.617.1) was first identified in India in December 2020, and L452R, E484Q, D614G, and P681R were detected as peak mutations that also affect antibody neutralization and transmission (19).

- The lota variant (B.1.526) was first identified in the United States in December 2020, using D614G, E484K, and A701V as peak mutations that affect antibody neutralization (19).

- The Zeta mutant (P.2) was first identified in Brazil in January 2021, and E484K and D614G were detected as spike mutations affecting antibody neutralization (19).

- Not all of the above variants have been shown to cause more severe illness (19).

\section{Booster dose}

There are several reasons why a booster dose of the COVID-19 vaccine may be needed.

i. Over time, your defenses against infections and illnesses, especially severe illnesses, decrease (that is, your immunity decreases). 
ii. Reduced protection against Variant of Concern (VOC)

iii. There is insufficient protection from the currently recommended prime series for some risk groups that may lack evidence from Phase 3 clinical trials.

The foundation for booster doses may vary by vaccine product, epidemiological situation, vaccination rate, and risk group. Still, in situations where global vaccine supply limitations continue, booster doses increase demand and are in short supply. It exacerbates inequality by consuming the supply it has. In national or local situations, you have not yet received a series of primary vaccinations. For now, the focus is on increasing global vaccination rates in the primary series (one or two doses for current EUL vaccines) (20).

\section{Fungal pandemic}

- The current rise of coronavirus disease (COVID-19) is associated with reports of fungal infections such as aspergillosis and mucormycosis, particularly among seriously ill patients treated with steroids. Recent cases of COVID-19 in India during the second wave of the pandemic are associated with increased reports of invasive mucormycosis after COVID-19 (21).

- Several factors can contribute to the development of mucormycosis in COVID-19 patients, including diabetes mellitus, corticosteroid use, obesity, and the progress of cytokine storms. SARS-CoV-2 triads, steroids, and unrestrained diabetes mellitus contributed to a significant increase in the incidence of vascular infiltrative maxillofacial zygomycosis. However, the existence of spores and other factors can also play a role (22).

- With an increasing number of critically ill patients infected with the ongoing COVID-19 pandemic and SARS-CoV-2, mucormycosis, prevalence Risk for patients at risk of mucormycosis based on the epidemiological burden of diabetes is essential to develop a base approach. Intrinsic, severity of COVID-19 disease, and use of immunomodulators, including the combination of corticosteroids and immunosuppressants in cancer and transplant patients (21).

The COVID-19 immunizations, as of now being affirmed or developed, are required to give probably some protection against new variations of the virus, as these antibodies get an expansive invulnerable reaction, including the scope of antibodies and cells. Consequently, changes or transformations in the infection ought not to deliver antibodies incapable. If any of these immunizations end up being less potent against at least one variation, the creation of the antibodies can be changed to secure against these variations $(1,23-24)$

Scaling up vaccine production and introducing vaccines as widely and quickly as possible will also be important ways to protect people before they are directly exposed to the virus and the risk of new variants emerging. Priority should be given to vaccinating high-risk populations everywhere to maximize global protection against new variants and minimize the risk of transmission. Moreover, ensuring equitable access to COVID-19 vaccines is more critical than ever in tackling the evolving pandemic. We expect that as more people are vaccinated, the circulation of the virus will decrease, resulting in fewer mutations. 
Developing countries lack the equipment to identify new options, even if they do occur. Given the strange outbreaks of death and virus infections, it is highly likely that new options and old methods of safe storage, treatment and infection are already emerging. Vaccines are not $100 \%$ operational. These new variants could threaten not only these developing and developing countries but other countries as well, as a new virus can move without worrying about borders, infect the entire world again and trigger a new pandemic that could easily threaten human survival. $(24,25)$.

\section{Conclusion}

Appropriate public health policy should take into account the emergence of any new viral variant. Moreover, more research is needed to cope with new variants of the virus. Current countermeasures should never be abandoned, including travel bans, nationwide quarantines, strict social distancing, especially during the holidays, frequent hand washing and respiratory hygiene. Characterization of any new viral variant requires molecular biology experiments, contact tracing and testing for COVID-19. Organizations like the WHO or CDC must track the Covid-19 mutation not only in the US and other European countries and in developing countries so that they are prepared for any mutation and stay one step ahead of the virus. Moreover, the vaccine should be prepared against new variants to get desirable results.

\section{Declarations}

\section{Ethics approval and consent to participate: Nil}

Consent for publication: all authors have consent for publication in journal.

Availability of data and material: it is mentioned above in the aspect of material.

Competing interests: Nil

Funding: Nil

Authors' contributions: M. D. have drafted the work and Sh. Gh. s ubstantively revised it. We both had substantial contributions to the conception of the idea of manuscript.

\section{Acknowledgements: Nil}

\section{References}

1. https://www.who.int/news-room/feature-stories/detail/the-effects-of-virus-variants-on-covid-19vaccines?gclid=Cj0KCQjwyZmEBhCpARIsALIzmnIfdtG9UazfQ_UfGAGsaLJcwJ-L9dwiY2XkberHCjVipoV9pJMTclaAv-OEALw_wcB

2. https://www.cdc.gov/coronavirus/2019-ncov/science/science-briefs/scientific-brief-emergingvariants.html 
3. Horby P, Huntley C, Davies N, et al. NERVTAG note on B.1.1.7 severity. SAGE meeting report. January $21,2021$.

4. Wu K, Werner AP, Molina JI, et al. mRNA-1273 vaccine induces neutralizing antibodies against spike mutants from global SARS-CoV-2 variants. bioRxiv. Posted January 25, 2021.

5. Xie X, Zou J, Fontes-Garfias CR, et al. Neutralization of N501Y mutant SARS-CoV-2 by BNT162b2 vaccine-elicited seraexternal icon. bioRxiv. Posted January 7, 2021. Greaney AJ, Loes AN, Crawford $\mathrm{KHD}$, et al. Comprehensive mapping of mutations to the SARS-CoV-2 receptor-binding domain that affect recognition by polyclonal human serum antibodies. bioRxiv. [Preprint posted online January 4, 2021]

6. Weisblum Y, Schmidt F, Zhang F, et al. Escape from neutralizing antibodies by SARS-CoV-2 spike protein variants external icon. eLife 2020;9:e61312.

7. Resende PC, Bezerra JF, de Vasconcelos RHT, at al. Spike E484K mutation in the first SARS-CoV-2 reinfection case confirmed in Brazil, 2020. [Posted on virological. or external icon on January 10 , 2021]

8. Cascella M, Rajnik M, Aleem A, Dulebohn SC, Di Napoli R. Features, Evaluation, and Treatment of Coronavirus (COVID-19). In: StatPearls. Treasure Island (FL): StatPearls Publishing; July 30, 2021.

9. Lopez Bernal J, Andrews N, Gower C, et al. Effectiveness of Covid-19 Vaccines against the B.1.617.2 (Delta) Variant. N Engl J Med. 2021;385(7):585-594. doi:10.1056/NEJMoa2108891

10. European Centre for Disease Prevention and Control. Threat assessment brief: the emergence of SARS-CoV-2 B.1.617 variants in India and situation in the EU/EEA. May 11, 2021. (https://www.ecdc.europa.eu/en/publications-data/threat-assessment-emergence-sars-cov-2-b1617variants).

11. Li Q, Wu J, Nie J, et al. The impact of mutations in SARS-CoV-2 spike on viral infectivity and antigenicity. Cell 2020;182(5):1284.e9-1294.e9.

12. Johnson BA, Xie $X$, Kalveram B, et al. Furin cleavage site is key to SARS-CoV-2 pathogenesis. August 26, 2020. (https://www.biorxiv.org/content/10.1101/2020.08.26.268854v1). preprint.

13. Romero PE, Dávila-Barclay A, Salvatierra G. The emergence of SARS-CoV-2 variant lambda (C.37) in South America. medRxiv. 2021

14. Tada T, Zhou H, Dcosta BM, Samanovic MI, Mulligan MJ, Landau NR. SARS-CoV-2 lambda variant remains susceptible to neutralization by mRNA vaccine-elicited antibodies and convalescent serum. bioRxiv. 2021

15. Acevedo ML, Alonso-Palomares L, Bustamante A. Infectivity and immune escape of the new SARSCoV-2 variant of interest Lambda. medRxiv. 2021

16. https://www.who.int/publications/m/item/weekly-epidemiological-update-on-covid-19--31-august2021

17. Messali, S., Bertelli, A., Campisi, G., Zani, A., Ciccozzi, M., Caruso, A. and Caccuri, F. (2021), A cluster of the new SARS-CoV-2 B.1.621 lineage in Italy and sensitivity of the viral isolate to the BNT162b2 vaccine. J Med Virol. https://doi.org/10.1002/jmv.27247 
18. Snell LB, Cliff PR, Charalampous T, et al. Rapid genome sequencing in hospitals to identify potential vaccine-escape SARS-CoV-2 variants [published online ahead of print, 2021 Aug 13]. Lancet Infect Dis. 2021;S1473-3099(21)00482-5. doi:10.1016/S1473-3099(21)00482-5

19. Mohammadi $M$, Shayestehpour M, Mirzaei $H$. The impact of spike mutated variants of SARS-CoV2 [Alpha, Beta, Gamma, Delta, and Lambda] on the efficacy of subunit recombinant vaccines [published online ahead of print, 2021 Aug 17]. Braz J Infect Dis. 2021;25(4):101606. doi:10.1016/j.bjid.2021.101606

20. https://www.who.int/news/item/10-08-2021-interim-statement-on-covid-19-vaccine-booster-doses

21. Al-Tawfiq JA, Alhumaid S, Alshukairi AN, et al. COVID-19 and mucormycosis superinfection: the perfect storm [published online ahead of print, 2021 Jul 24]. Infection. 2021;1-21. doi:10.1007/s15010-021-01670-1

22. Skoda A, Pavleas I, Drogari-Apiranthitou M. Epidemiology and diagnosis of mucormycosis: an update. J Fungi. 2020;6:1-20. DOI: 10.3390/jof6040265.

23. Macera M, De Angelis G, Sagnelli C, Coppola N, Vanvitelli C-G. Clinical presentation of COVID-19: Case series and review of the literature. Int J Environ Res Public Health. 2020;17(14):5062.

24. Cucinotta D, Vanelli M. WHO declares COVID-19 a pandemic. Acta Biomed. 2020;91(1):157-160. 25. AJMC Staff. A timeline of COVID-19 developments in 2020: AJMC; 2020 [updated 25 November 2020.

\section{Figures}




\section{Share of Population That Has Received At Least One Dose by Region}

- African Region - Eastern Mediterranean Region - European Region

- Region of the Americas — South-East Asia Region — Western Pacific Region

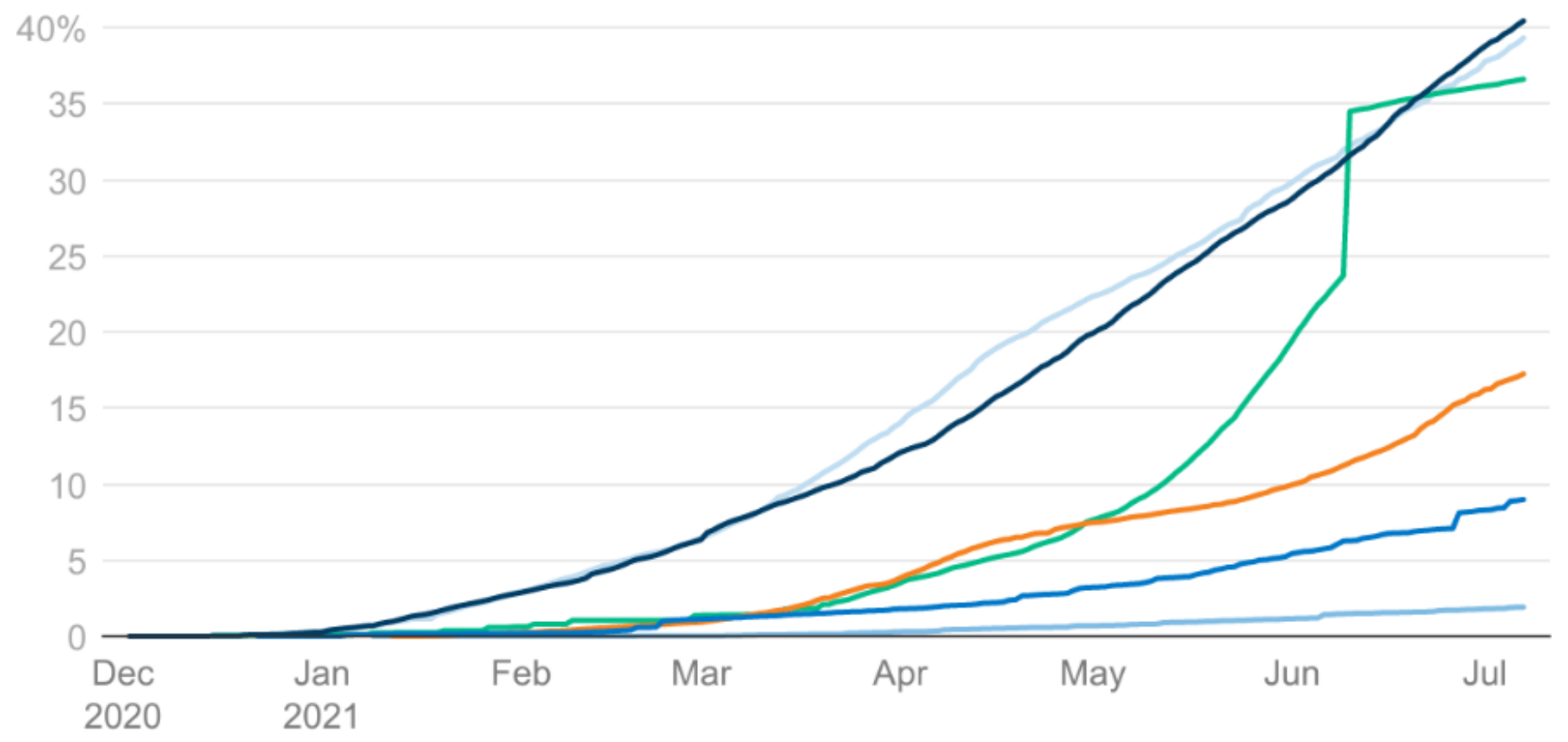

NOTE: Data as of July 7, 2021 to account for lag in country reporting. On June 10, 2021, China reported for the first time the number of people who have received at least one dose, resulting in a sharp increase from our lower-bound calculations.

SOURCE: Our World in Data, WHO, United Nations

\section{Figure 1}

Percentage of the population receiving at least one dose per region, courtesy of Our World in Data, WHO, United Nations, and kff. 